\title{
Computed tomographic features of Scottish Fold osteochondrodysplasia
}

\author{
Seyoung Lee, Juyeon Oh, Jihye Choi* \\ Veterinary Medical Imaging, College of Veterinary Medicine and BK 21 Plus Project Team, Chonnam National University, \\ Gwangju 61186, Korea
}

\begin{abstract}
An 18-month-old, Scottish Fold with severe lameness and pain in the fore- and hind-limbs was diagnosed as Scottish Fold osteochondrodysplasia based on radiography showing mis-shapened phalanges, bone formation around the tarsal and metatarsal joints, joint space narrowing, and ankylosis. Although computed tomography (CT) is not considered a primary method for examining Scottish Fold osteochondrodysplasia, CT was undertaken in this case to investigate the cause of pain and reluctant ambulation in the forelimbs. CT showed immaturely ossified bone proliferation in the carpal bones. CT-based multiplanar reconstruction can reveal the anatomic relationship between the bone lesion and the joint in various directions.
\end{abstract}

Key words: cat, CT, radiography, Scottish Fold osteochondrodysplasia

*Corresponding author

Jihye Choi

Veterinary Medical Imaging, College of Veterinary Medicine and BK 21 Plus Project Team, Chonnam National University, 77 Yongbong-ro, Buk-gu, Gwangju 61186, Korea

Tel: $+82-62-530-2821$

Fax: +82-62-530-2809

E-mail: imsono@jnu.ac.kr

ORCID:

Seyoung Lee

https://orcid.org/0000-0001-9236-7309

Juyeon Oh

https://orcid.org/0000-0002-6301-3947

Jihye Choi

https://orcid.org/0000-0002-1258-7158

Conflict of Interest

The authors declare no conflicts of interest.

Received: July 10, 2019

Revised: October 8, 2019

Accepted: February 24, 2020
Scottish Fold osteochondrodysplasia (SFOCD) is a genetic disorder characterized by the presence of an autosomal dominant homozygous gene for folded ear ( $F d$ gene) in Scottish Fold cats [1-3]. The $F d$ gene has been associated with cartilage deformity, although the exact etiology of SFOCD is unknown [2,4]. Defective cartilage formation leads to various degrees of osteoarthropathy, including osteophytosis, exostosis, bony ankyloses, and progressive osteoarthritis [2]. The tissues involved in exostosis include the hypertrophic hyaline cartilage layer, as well as necrotic cartilage and fibrotic or fragmented cartilage; proliferating and resting chondrocytes are the main cell types observed in such tissues because chondrocytes do not fully mature in exostotic tissues [1-2,5].

SFOCD is usually diagnosed based on the combination of breed type, clinical signs, and radiographic results. Radiographic changes are mainly observed in distal limbs and caudal vertebrae and include exostosis, osteoarthritis around the affected joints, and defective conformation in the phalanges [6,7]. Bony ankyloses and progressive osteoarthritis can cause pain, lameness, and gait abnormalities in affected cats, even though some animals may be asymptomatic [2]. In most cases, the severity of the bony changes is correlated with the clinical signs [8].

Computed tomography (CT) produces cross-sectional images of variable thickness that can be viewed without superimposition. Compared to radiography, CT can more accurately discriminate between tissues and can separate soft tissues and bone structures based on signal attenuation. In this case report, bone and joint lesions are investigated by examining the results of conventional radiography and CT study in a cat with a severe form of SFOCD.

An 18-month-old, $1.9 \mathrm{~kg}$, spayed female Scottish Fold cat was presented at the Chonnam National University Veterinary Teaching Hospital for reluctant ambulation and severe lameness. Intermittent hindlimb lameness began at 6 months old and progressed and worsened in spite of the administration of non-steroidal anti-inflammatory drugs. Recently, the cat appeared to be in pain originating from the carpal joint areas and had stopped walking. Physical examination revealed swelling in the tarsal regions, and the cat, overwhelmed by pain, cried during palpation and had difficulty bearing weight on the joints. The cat also appeared to be in pain during palpation of the carpal joints, although to a lower extent than that during palpation of the tarsal joints.

Lateral and anterior-posterior radiographs of the fore- and hind-limbs, 

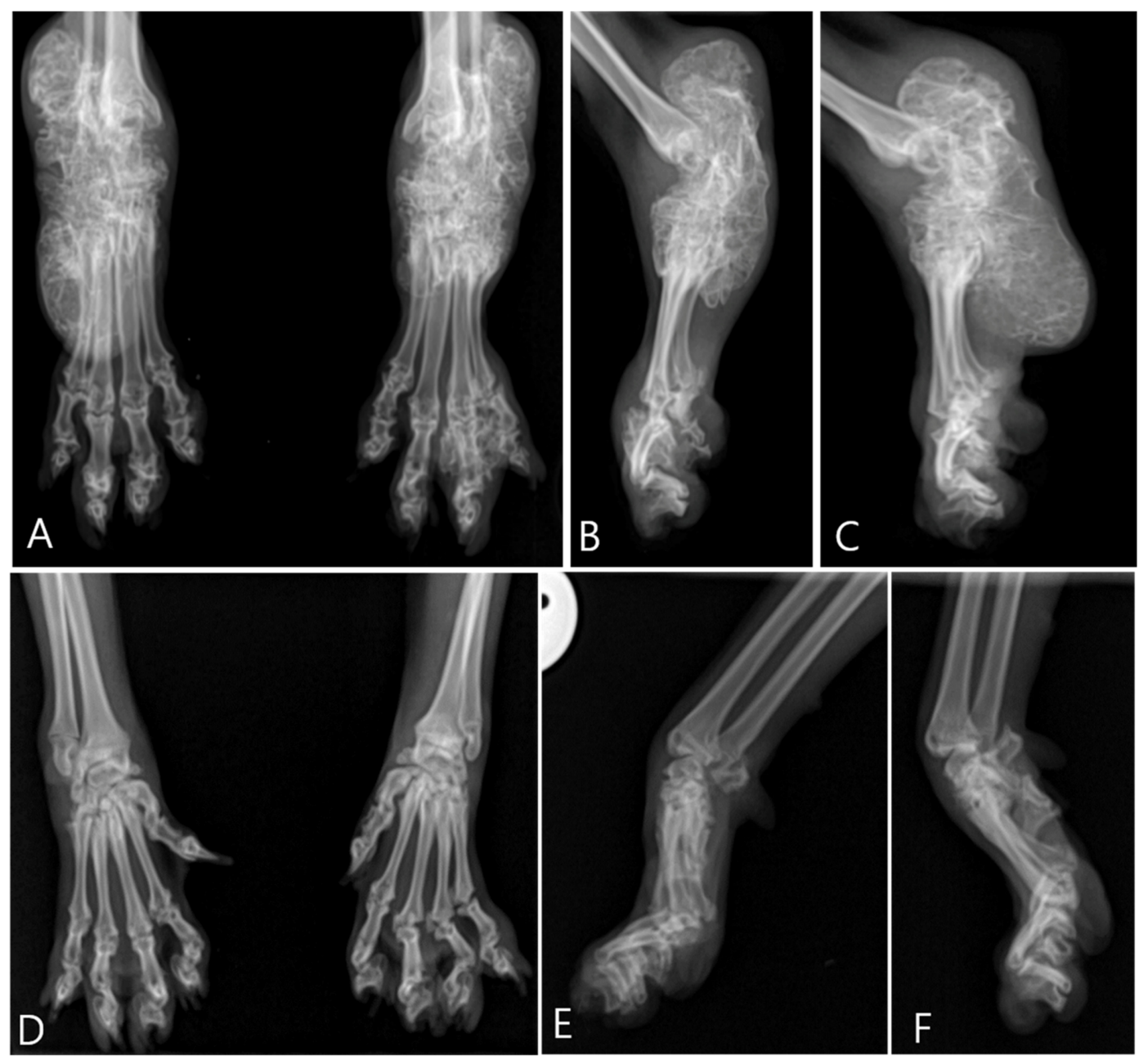

Fig. 1. Radiographs of bilateral distal hindlimbs (A-C) and distal forelimbs (D-F). On dorsopalmar radiograph (A) of the hindlimbs, exostoses showing internal hypodense and outer hyperdense patterns resulted in ankylosis of the bilateral tarsal joint. The margin of the new bone was smooth. Lateral views of right (B) and left (C) hindlimbs revealed that more severe exostosis grew along the left metatarsal joint in the posterior direction. It was difficult to distinguish between the talus and calcaneus and to recognize the margins of the tibia and fibula due to exostoses. All metatarsal bones were shortened. Note, mild exostosis in the third right phalange bone on the dorsopalmar view. No significant deformity was observed on the dorsopalmar radiograph (D) of the carpal region or on lateral views of the right $(\mathrm{E})$ and left $(\mathrm{F})$ carpal regions. Left side of the image is the right limb, and right side is the left limb on the dorsopalmar radiographs.

including phalanges, were obtained (Fig. 1). Extensive exostoses were detected in the bilateral tarsal joints and were particularly prominent caudal to the calcaneus. The proliferative bone masses consisted of an internal hypodense area and an outer hyperdense area with a smooth margin. The masses were approximately $2.4 \times 5.1 \mathrm{~cm}$ in the right tarsal region and $2.5 \times 6.1 \mathrm{~cm}$ in the left tarsal region. Severe exostosis had narrowed the bilateral tarsal joint spaces and induced tarsal ankylosis. All metatarsal bones and phalanges were shorter than normal and showed irregular margins due to osteophytosis, particularly from the third and fourth right distal phalange bone. No significant deformities were observed in the forelimbs, including their carpal joints and extremities. Based on the cat's breed, clinical signs, and radiographic findings, SFOCD was diagnosed.
The cat also underwent CT examination to investigate the cause of pain and the lack of weight-bearing behavior in the forelimbs. CT imaging was performed using a 16-row multidetector CT scanner (Somatom Emotion, Siemens Medical Systems, Forchheim, Germany) with settings of $200 \mathrm{mAs}$, $130 \mathrm{kV}$, and $1 \mathrm{~mm}$ slice thickness for the vertebrae and 270 $\mathrm{mAs}, 130 \mathrm{kV}$, and $1 \mathrm{~mm}$ slice thickness for the fore- and hind-limbs. The acquired transverse CT images were reformatted into sagittal and dorsal planes and underwent threedimensional (3D) volume rendering. All CT images were evaluated in both the bone window (window level: 450 Hounsfield Units [HU]; window width: 1,500 HU) and the soft tissue window (window level: $40 \mathrm{HU}$; window width: $400 \mathrm{HU})$.

On CT images of hindlimbs, severe amorphous exostoses 


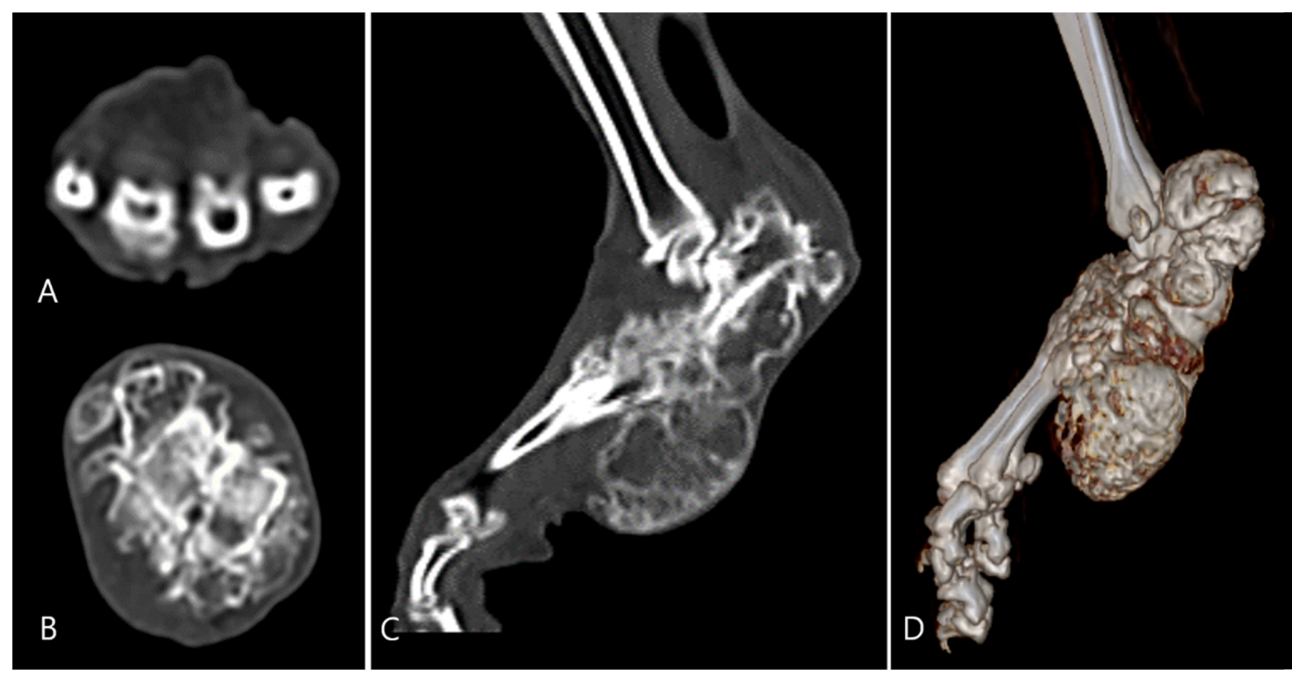

Fig. 2. Computed tomography images of the left hindlimb. On transverse plane (A) of left metatarsal bones, bone spurs were detected. On transverse (B) and sagittal (C) planes of left calcaneus and talus, the margins of the calcaneus and talus were not discernible and irregular in the new bone. The cortex of the proliferative bone was hyperdense, and the medulla was hypodense. New bone proliferated in the direction of the posterior and metatarsal bones. The severe proliferative bone mass was more distinctly observed on $3 \mathrm{D}$ volume rendering reconstruction (D).

comprising outer hyperattenuating and inner hypoattenuating compositions were noted in the right calcaneus and talus (Fig. 2). The exostosis in the right tarsal region was severe in the distal talus and calcaneus bones and the new bone formed by the exostosis in the right fourth tarsal bone was larger than the diameter of the fourth tarsal bone and had an indistinct border. Mild osteophyte areas were observed in the distal fibula and tibia. Exostosis was detected extending from the left calcaneus to one-third of the proximal metatarsal bone, and the fourth tarsal and central tarsal bones were merged by a proliferative bone lesion with an indistinct boundary. The volume of the left tarsal joint capsule was reduced by osteophytes of the proximal metatarsal bone. Outer hyperattenuating and inner hypoattenuating bone proliferation areas were detected at the fourth proximal and middle phalanges. The $3 \mathrm{D}$ volume rendering performed on transverse images of the hindlimbs revealed a severe proliferative bone mass with an irregular margin (Fig. 2); from the calcaneus to the metatarsal bone.

On the carpal CT images, hypoattenuating osteophytes were noted in the radial, ulnar, and accessory carpal bones, and the margins of the bilateral metacarpal bones were obscured by proliferative osteophytes (Fig. 3). The proliferative osteophytes consisted of outer hyperattenuating and inner hypoattenuating composition. Mild osteophytes of the phalanges were also identified. No significant deformities were observed at the vertebrae except for an overall presence of mild bony spurs at the caudal vertebrae. The CT study results indicated the severity and location of the cat's bone and joint lesions suggesting that the animal's forelimb lameness and pain were consequences of SFOCD of the carpal joints and bones.
This study described several CT features of SFOCD, including short mis-shapened phalanges, irregularly shaped tarsal, carpal, metatarsal, and metacarpal bones due to proliferative bone formation, obscured bone margins and narrowed joint spaces, massive bone masses (exostoses) in the distal limbs, and changes in vertebrae. This case of SFOCD was a severe form of the disease, and tarsal joint ankyloses had developed.

SFOCD is clinically diagnosed based on radiographic findings [2,9]. In this case, typical radiographic findings, including thickening of the shortened phalanges, osteophytes at distal limb joints, and exostosis, were most prominent in distal limb areas, particularly at the tarsometatarsal joints rather than at the carpal or metacarpal joints. Lesion locations were compatible with those reported in previous cases $[2,5,8,9]$. The bone density of the tarsometatarsal joints was heterogeneous, even though there were no lytic changes, because new bone formed by osteophytosis and exostosis was superimposed on pre-existing bone structures. Exostoses formed a massive bony area around the joints, consisting of outer radiopaque and inner radiolucent regions. This characteristic radiographic finding has been previously described as having a "moth-eaten appearance" [2,5,8,9]. Ankyloses of the tarsal joint had developed. When Scottish Fold cats possessing the $F d$ gene are older than 7 weeks, typical radiographic lesions indicative of SFOCD can be seen even in the absence of clinical signs in cats with a normal gait [2,9]. Thus, regardless of the presence of clinical signs, radiography is considered a useful diagnostic tool for detecting SFOCD in cats.

However, radiographic findings were insufficient to explain the forelimb pain in this case. Radiography has some limitations when investigating the joints of small breed dogs or 

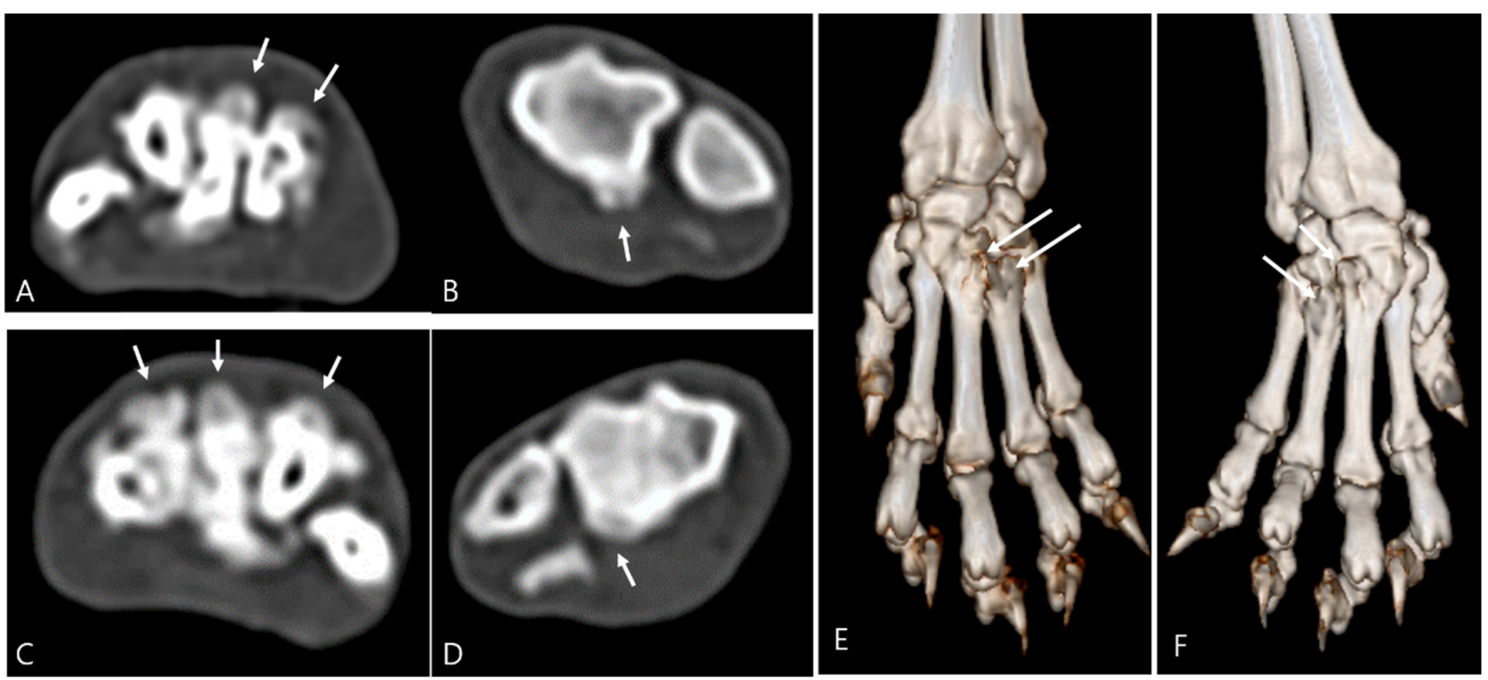

Fig. 3. CT images of the distal forelimbs. Transverse CT images revealed hypodense bony spurs (arrows) in right metacarpal bones (A), right ulna and radius (B), left metacarpal bones (C), and the left ulna and radius (D). The proliferative bone had an irregular margin and was hypodense compared to the cortex of normal bone; the proliferative bone had fused with adjacent bones. Irregular changes (arrows) in the metacarpal bones as a result of bone proliferation were observed in the 3rd and 4th digits on 3D volume rendering reconstruction $(\mathrm{E}, \mathrm{F})$. CT, computed tomography.

cats, in detail, because overlapping between the lesion and adjacent structures can hinder the ability to define the exact location of any newly formed bone and disrupt the delineation of the lesion's margin. Lesion discrimination is further limited because carpal and tarsal joints consist of numerous small bones and narrow joint spaces.

Compared to radiographic images, CT images created by performing multiplanar reformation (MPR) show joint and bone lesions in more detail. In the tarsal joint, mildly proliferated, hypodense lesions, which had not been detected on radiographs, were observed in tarsal bone, tibia, fibula, and coccygeal vertebrae areas on CT images. Following 3D volume rendering of the CT images, the exostosis exhibited an irregular surface which seemed to be associated with the pain induced in the hindlimb. In the carpal and metacarpal joints, mildly proliferated, hypodense lesions induced narrowing of the joint spaces and pain. These hypodense lesions were small and had lower densities than those of the adjacent bone structures; thus they were suspected as chondromatous proliferations, immaturely ossified. They were not detected on radiography due to their small size and lack of mineral density.

The CT features of SFOCD are rarely reported because many cats with SFOCD do not show clinical signs during the early stage or even over the entire course of the disease, and characteristic radiographic findings are usually observed in symptomatic cats [2]. However, when radiographic findings cannot fully explain the clinical signs exhibited by the patient, such as in this case, CT examination is needed to determine the location and extent of the lesion(s) [6].

The radiographic and CT features of SFOCD have been described in this report. Bone changes in the carpal joint and vertebrae that were not detected by radiography were observed on CT images, and the CT observations helped explain the forelimb pain of the patient. Transverse CT images clearly displayed immaturely ossified proliferative changes in the affected bone as well as the presence of osteophytosis and exostosis; all of these changes related to the clinical signs. The MPR images were particularly helpful for investigating the relationship between bone lesions and joint spaces in various directions, and $3 \mathrm{D}$ volume rendering revealed the surface of the new bone. Although CT examination is not essential for diagnosing SFOCD, this modality can be used to provide additional information about the location and extent of a lesion, particularly in patients with unremarkable radiographic findings.

\section{Acknowledgements}

This research was supported by the Basic Science Research Program of the National Research Foundation (NRF) of South Korea, which is funded by the Ministry of Science, ICT, and Future Planning (2018R1A2B6006775).

\section{References}

1. Allan G. Radiographic Signs of Joint Disease in Dogs and Cats. In: Thrall DE (ed.). Textbook of Veterinary Diagnostic Radiology. 6th ed. pp. 339-340, Saunders Elsevier, St. Louis, 2013.

2. Jack OF. Congenital bone lesions in cats with folded-ears. Bull Fel Advis Bur 1975;14:2-4.

3. Kinns J, Malinowski R, McEvoy F, Schwarz T, Zwingenberger A. Special Software Applications. In: Schwarz T, Saunders J (eds.). Veterinary Computed Tomography. p. 73, Wiley Blackwell, New Jersey, 2011. 
4. Harasen GL, Little SE. Mucoskeletal Diseases. In: Little S (ed.). The Cat: Clinical Medicine and Management. p. 713, Elsevier, St. Louis, 2012.

5. Hubler M, Volkert M, Kaser-Hotz B, Arnold S. Palliative irradiation of Scottish Fold osteochondrodysplasia. Vet Radiol Ultrasound 2004;45:582-585.

6. Chang J, Jung J, Oh S, Lee S, Kim G, Kim H, Kweon O, Yoon J, Choi M. Osteochondrodysplasia in three Scottish Fold cats. J Vet Sci 2007;8:307-309.

7. Malik R, Allan GS, Howlett CR, Thompson DE, James G,
McWhirter C, Kendall K. Osteochondrodysplasia in Scottish Fold cats. Aust Vet J 1999;77:85-92.

8. Takanosu M, Takanosu T, Suzuki H, Suzuki K. Incomplete dominant osteochondrodysplasia in heterozygous Scottish Fold cats. J Small Anim Pract 2008;49:197-199.

9. Pollard RE, Wisner ER. Orthopedic Diseases of Young and Growing Dogs and Cats. In: Thrall DE (ed.). Textbook of Veterinary Diagnostic Radiology. 6th ed. p. 277, Saunders Elsevier, St. Louis, 2013. 